\title{
Anatomia das folhas, brácteas e escapos de Actinocephalus (Koern.) Sano (Eriocaulaceae)
}

\author{
ALINE ORIANI ${ }^{1,3}$, VERA LUCIA SCATENA $^{1}$ e PAULO TAKEO SANO ${ }^{2}$
}

(recebido: 19 de setembro de 2003; aceito: 9 de dezembro de 2004)

\begin{abstract}
Anatomy of the leaves, bracts and scapes of Actinocephalus (Koern.) Sano (Eriocaulaceae)). The anatomy of the leaves, bracts and scapes of 20 species (41 specimens) of Actinocephalus from the "campos rupestres" of Brazil were studied. Our aim was the characterization of this new genus in an anatomical basis, besides elucidating some ecological aspects of the group. The leaves and bracts are similar, presenting an uniseriate epidermis, with elongated cells in longitudinal orientation; stomata on the abaxial surface, with substomatal chambers; loosely aggregated chlorenchyma; collateral vascular bundles enclosed in a double sheath, and parenchymatous bundles sheath extensions. The scapes present an uniseriate epidermis; substomatal chambers; loosely aggregated chlorenchyma; discontinuous endodermis; sinuous pericycle and collateral vascular bundles. The presence of elongated cells in longitudinal orientation in the epidermis as well as in the vascular bundles sheath extensions in the leaves and bracts, besides discontinuous endodermis in the scapes and substomatal chambers in the three organs studied are consistent characters for the genus. The anatomical characteristics of the organs studied are mesomorphic.
\end{abstract}

Key words - Actinocephalus, anatomy, bracts, leaves, scapes

RESUMO - (Anatomia das folhas, brácteas e escapos de Actinocephalus (Koern.) Sano (Eriocaulaceae)). Estudou-se a anatomia das folhas, brácteas e escapos de 20 espécies (41 espécimes) de Actinocephalus, que ocorrem nos campos rupestres do Brasil, visando caracterizar anatomicamente o novo gênero proposto para a família, além de elucidar alguns aspectos ecológicos do grupo. As folhas e as brácteas são anatomicamente semelhantes entre si e apresentam epiderme unisseriada, com células alongadas no sentido longitudinal; estômatos na face abaxial, com câmaras subestomáticas; parênquima clorofiliano frouxo; feixes vasculares colaterais envolvidos por bainha dupla e extensão de bainha dos feixes constituída por células parenquimáticas alongadas. Os escapos apresentam epiderme unisseriada; câmaras subestomáticas; parênquima clorofiliano frouxo; endoderme descontínua; periciclo sinuoso e feixes vasculares colaterais. A presença de células alongadas tanto na epiderme como nas extensões de bainha dos feixes vasculares nas folhas e nas brácteas, assim como endoderme descontínua nos escapos e câmara subestomática nos três órgãos estudados são caracteres consistentes para o gênero. As características anatômicas dos órgãos estudados são mesomórficas.

Palavras-chave - Actinocephalus, anatomia, brácteas, escapos, folhas

\section{Introdução}

Eriocaulaceae apresenta 10-11 gêneros e cerca de 1.200 espécies, com distribuição pantropical (Giulietti \& Hensold 1990, Sano 2004). Habitam predominantemente os terrenos brejosos das regiões tropicais e subtropicais de todo o mundo, sendo o seu maior centro de diversidade genética a região tropical da América do Sul, especialmente o Brasil (Joly 1993).

As Eriocaulaceae são amplamente distribuídas nos campos rupestres brasileiros, principalmente na Cadeia

\footnotetext{
1. Universidade Estadual Paulista, Instituto de Biociências, Departamento de Botânica, Caixa Postal 199, 13506-900 Rio Claro, SP, Brasil.

2. Universidade de São Paulo, Instituto de Biociências, Departamento de Botânica, Caixa Postal 11461, 05422-970 São Paulo, SP, Brasil.

3. Autor para correspondência: aoriani@ terra.com.br
}

do Espinhaço, que se estende ao longo dos estados de Minas Gerais e Bahia (Giulietti \& Pirani 1988). Mais de $90 \%$ das espécies pertencem aos gêneros Paepalanthus Mart., Syngonanthus Ruhl. e Eriocaulon L. (Splett et al. 1993). Paepalanthus é o maior gênero da família e apresenta ampla variabilidade morfológica (Scatena \& Moraes 1996).

Estudos anatômicos dos órgãos vegetativos de espécies de Eriocaulaceae eram restritos praticamente aos de Poulsen (1888), Holm (1901), Ruhland (1903), Malmanche (1919) e Tomlinson (1969). Nas décadas de 1980, 1990 e 2000, vários trabalhos foram publicados com espécies brasileiras de diferentes gêneros, incluindo: Paepalanthus (Castro \& Menezes 1990, 1995, Scatena \& Barros 1996, Scatena \& Moraes 1996, Scatena et al. 1998, Scatena \& Rosa 2001, Coan et al. 2002), Eriocaulon (Monteiro et al. 1984, Scatena et al. 1999a, Coan et al. 2002), Blastocaulon Ruhl. (Scatena et al. 1999b), Leiothrix Ruhl. (Monteiro et al. 1985, Scatena 
\& Rocha 1995, Scatena \& Giulietti 1996, Giulietti et al 1998, Coan et al. 2002), Syngonanthus (Scatena \& Menezes 1993, 1996, Coan et al. 2002), Tonina Aubl. e Philodice Mart. (Coan et al. 2002). Nesses trabalhos os autores discutiram a ocorrência de características anatômicas, relacionando-as com o ambiente e com a taxonomia do grupo.

O primeiro estudo cladístico para Eriocaulaceae foi proposto por Giulietti et al. (1995), apresentando Paepalanthus como um gênero polifilético. Uma análise mais recente apresentou uma árvore de consenso estrito para dados anatômicos e macromorfológicos, confirmou o polifiletismo de Paepalanthus e sugeriu que este gênero deveria ser dividido em vários pequenos grupos monofiléticos (Giulietti et al. 2000). Como resultado dessa análise, Sano (2004), a partir de Paepalanthus sect. Actinocephalus (Koern.) Ruhland, delimitou um novo gênero: Actinocephalus (Koern.) Sano, incluindo 29 espécies.

Considerando Eriocaulaceae como uma das famílias diagnósticas dos campos rupestres brasileiros, o objetivo deste trabalho foi caracterizar anatomicamente folhas, brácteas e escapos de Actinocephalus (Eriocaulaceae), visando corroborar ou não o novo gênero proposto por Sano (2004), além de elucidar aspectos ecológicos do grupo.

\section{Material e métodos}

O estudo anatômico de folhas, brácteas e escapos de Actinocephalus (Koern.) Sano foi feito em material obtido de exsicatas do Herbário do Departamento de Botânica da Universidade de São Paulo (SPF), provenientes de diferentes localidades do Brasil. Utilizou-se também material coletado em diferentes municípios de Minas Gerais, Brasil, cujas exsicatas estão depositadas no Herbário do Instituto de Biociências da Universidade Estadual Paulista (HRCB). Os espécimes estudados estão detalhados na tabela 1. De todas as espécies do gênero, apenas oito não foram estudadas por não ter sido possível a obtenção das mesmas, uma vez que se tratavam de holótipos ou se encontravam em herbários fora do país. Folhas, brácteas e escapos foram fervidos em água com glicerina para expansão dos tecidos e posteriormente transferidos para álcool $70 \%$. Foram feitas secções anatômicas transversais à mão livre, com lâminas de barbear, na região mediana das folhas, das brácteas e dos escapos, as quais foram submetidas ao processo de dupla coloração com fucsina e azul de astra (Roeser 1962) e montadas em lâminas semi-permanentes. Também foram feitas secções longitudinais na região mediana das folhas e das brácteas de A. ramosus (Wikstr) Sano e A. stereophyllus (Ruhland) Sano. Os resultados foram documentados por meio de desenhos organográficos e fotomicrografias, feitos com o auxílio de microscópio com câmara clara Olympus CBA e de fotomicroscópio Olympus BX40, respectivamente.

\section{Resultados}

Folhas e brácteas - As folhas e as brácteas dos diferentes espécimes estudados de Actinocephalus (Koern.) Sano, em secção transversal da região mediana, estão representadas nos diagramas das figuras 1-14. Todas as folhas estudadas apresentam o mesmo padrão anatômico (figuras 1-9), sendo este semelhante ao encontrado nas brácteas (figuras 10-14).

As margens das folhas e das brácteas apresentam parênquima clorofiliano logo abaixo da epiderme (figuras 1, 4-11, 14), ou, menos frequentemente, colênquima (figuras 2-3,12-13). O mesofilo é descontínuo devido à presença de extensões de bainha dos feixes vasculares maiores (figuras 1-14).

Os feixes vasculares são colaterais e envolvidos por bainha dupla em todas as folhas e brácteas estudadas (figuras 1-14). Apresentam-se em número variado, com disposição intercalada de feixes maiores e menores, e encontram-se dispostos num mesmo nível e equidistantes das epidermes (figuras 1-3, 5, 8, 10-14), ou distribuídos em séries de diferentes níveis (figuras 4, 6-7, 9). A presença de hipoderme foi observada somente em A. claussenianus (figuras 14, 17).

A epiderme é unisseriada, de forma alongada no sentido longitudinal, em secção transversal, na maioria das folhas (figuras 15-16, 18-20) e das brácteas (figuras 21-23), com exceção de A. claussenianus (figura 17). $\mathrm{O}$ espessamento da parede de suas células pode ser homogêneo, como em A. brachypus (figuras 15, 21), A. falcifolius (figura 19), A. polyanthus var. polyanthus (figuras 22-23), ou com a parede periclinal externa mais espessada que a interna, como em A. robustus (figura 16), A. claussenianus (figura 17), A. cabralensis (figura 18) e A. polyanthus var. bifrons (figura 20).

Os estômatos ocorrem somente na face abaxial, com câmaras subestomáticas amplas, em todas as folhas e brácteas dos espécimes estudados (figuras 15, 17, 20-21, 23). As câmaras subestomáticas são delimitadas por células epidérmicas que, em secção longitudinal, aparecem intercaladas com os estômatos e possuem a forma da letra " $\mathrm{T}$ " invertida, como pode ser observado em A. ramosus (figura 24, seta). Em secção transversal, essas células epidérmicas apresentam-se alongadas e curtas no nível dos estômatos, dependendo da altura em que foi feita a secção (figuras 20-21, 23). 
Tabela 1. Espécies e coleções utilizadas no estudo anatômico (CFCR: Coleção Flora dos Campos Rupestres; CFSC: Coleção Flora da Serra do Cipó, *Espécies novas em fase de descrição). s.l. - sem local; s.d. - sem data.

Table 1. Species and botanic material used in the anatomical study (CFCR: "Campos Rupestres" Flora Collection; CFSC: "Serra do Cipó" Flora Collection; * New species in description phase). s.l. = no place; s.d. = no date.

\begin{tabular}{|c|c|c|}
\hline Espécie & Localidade & Coleção \\
\hline \multirow{3}{*}{$\begin{array}{l}\text { Actinocephalus bongardii } \\
\text { (A.St.-Hil.) Sano }\end{array}$} & MG - Goveia & Giulietti et al. CFCR 1731 29-VIII-1981 \\
\hline & GO - Cocalzinho & Forzza et al. 872 28-V-1998 \\
\hline & MG - Diamantina & Scatena et al. 253 1-II-2002 \\
\hline \multirow[t]{4}{*}{ A. brachypus (Bong.) Sano } & MG - Diamantina & Giulietti et al. CFCR 2334 30-X-1981 \\
\hline & MG - Diamantina & Hensold et al. CFCR 2673 2-XII-1981 \\
\hline & MG - Diamantina & Mello-Silva et al. CFCR 8640 23-XI-1985 \\
\hline & MG - Diamantina & Braga \& Chaves 446 3-XII-1991 \\
\hline A. cabralensis (Silveira) Sano & MG - Augusto de Lima. Serra do Cabral & Sano et al. CFCR 15292 20-III-1994 \\
\hline A. callophyllus (Silveira) Sano & MG - Pico do Itambé & Silveira 504 IV-1908 \\
\hline \multirow[t]{3}{*}{ A. ciliatus (Bong.) Sano } & MG - Serro & Souza et al. 8342 11-III-1995 \\
\hline & MG - Costa Sena & Sano et al. 867 12-II-1998 \\
\hline & MG - Trinta Réis & Godoy et al. CFCR 11933 12-XII-1987 \\
\hline A. cipoensis (Silveira) Sano & s.l. & Arbo et al. 4940 \\
\hline A. claussenianus (Koern.) Sano & MG - Costa Sena. Morro do Catete & Silveira 481 \\
\hline A. denudatus (Koern.) Sano & s.l. & Giulietti et al. CFCR 1873 \\
\hline \multirow[t]{3}{*}{ A. diffusus (Silveira) Sano } & MG - Santana do Riacho & Pirani CFSC 5956 29-II-1980 \\
\hline & MG - Santana do Riacho & Esteves et al. CFSC 5988 14-XI-1984 \\
\hline & MG - Santana do Riacho & Giulietti CFSC 5642 15-VIII-1979 \\
\hline A. falcifolius (Koern.) Sano & s.l. & s.d. \\
\hline \multirow[t]{3}{*}{ A. herzogii (Moldenke) Sano } & BA - Piatã. Serra da Tromba & Ganev 285 14-V-1992 \\
\hline & BA - Piatã & Harley et al. 24189 14-II-1987 \\
\hline & BA - Piatã. Serra de Santana & Queiroz H51527 10-II-1992 \\
\hline A. nodifer (Silveira) Sano & MG - Chapada do Couto & Silveira 684 IV-1918 \\
\hline \multirow{4}{*}{$\begin{array}{l}\text { A. polyanthus var. bifrons (Silveira) } \\
\text { Sano }\end{array}$} & MG - Diamantina & Sano et al. 796 11-XII-1997 \\
\hline & & \\
\hline & MG - Diamantina & Scatena et al. 244 31-I-2002 \\
\hline & MG - Diamantina & Scatena et al. 248 1-II-2002 \\
\hline \multirow{5}{*}{$\begin{array}{l}\text { A. polyanthus var. polyanthus } \\
\text { (Bong.) Sano }\end{array}$} & PR - Ponta Grossa & Krieger 14362 19-XII-1976 \\
\hline & SP - Piquete & Sano et al. 142 5-VI-1995 \\
\hline & MG - Diamantina & Sano et al. 815 11-II-1998 \\
\hline & MG - Santana do Riacho. Serra do Cipó & Scatena et al. 236 29-I-2002 \\
\hline & MG - Santana do Riacho. Serra do Cipó & Scatena et al. 237 29-I-2002 \\
\hline \multirow[t]{3}{*}{ A. ramosus (Wikstr) Sano } & RJ - Massambaba & Fontella et al. $3198 \quad 27-\mathrm{X}-1993$ \\
\hline & MG - Santana do Riacho. Serra do Cipó & Sano et al. 544 20-X-1997 \\
\hline & MG - Diamantina & Scatena et al. 249 1-02-2002 \\
\hline A. rigidus (Bong.) Sano & MG - Diamantina & Sano et al. 691 8-XII-1997 \\
\hline A. robustus (Silveira) Sano & MG - Santana do Riacho. Serra do Cipó & Sano et al. 657 23-X-1997 \\
\hline \multirow[t]{2}{*}{ A. stereophyllus (Ruhland) Sano } & MG - Diamantina & Sano et al. 705 8-XII-1997 \\
\hline & MG - Diamantina & Scatena et al. 252 1-II-2002 \\
\hline Actinocephalus sp. nov. 1 Sano* & MG - Botumirim & M.G.C. \& S.T.S. 479 25-VII-1991 \\
\hline Actinocephalus sp. nov. 2 Sano* & SE - Serra de Itabaiana & Andrade-Lima SPF 30379 20-IV-1974 \\
\hline Actinocephalus sp. nov. 3 Sano* & MG - Rio Vermelho & Mello-Silva et al. CFCR 10240 9-IX-1986 \\
\hline
\end{tabular}




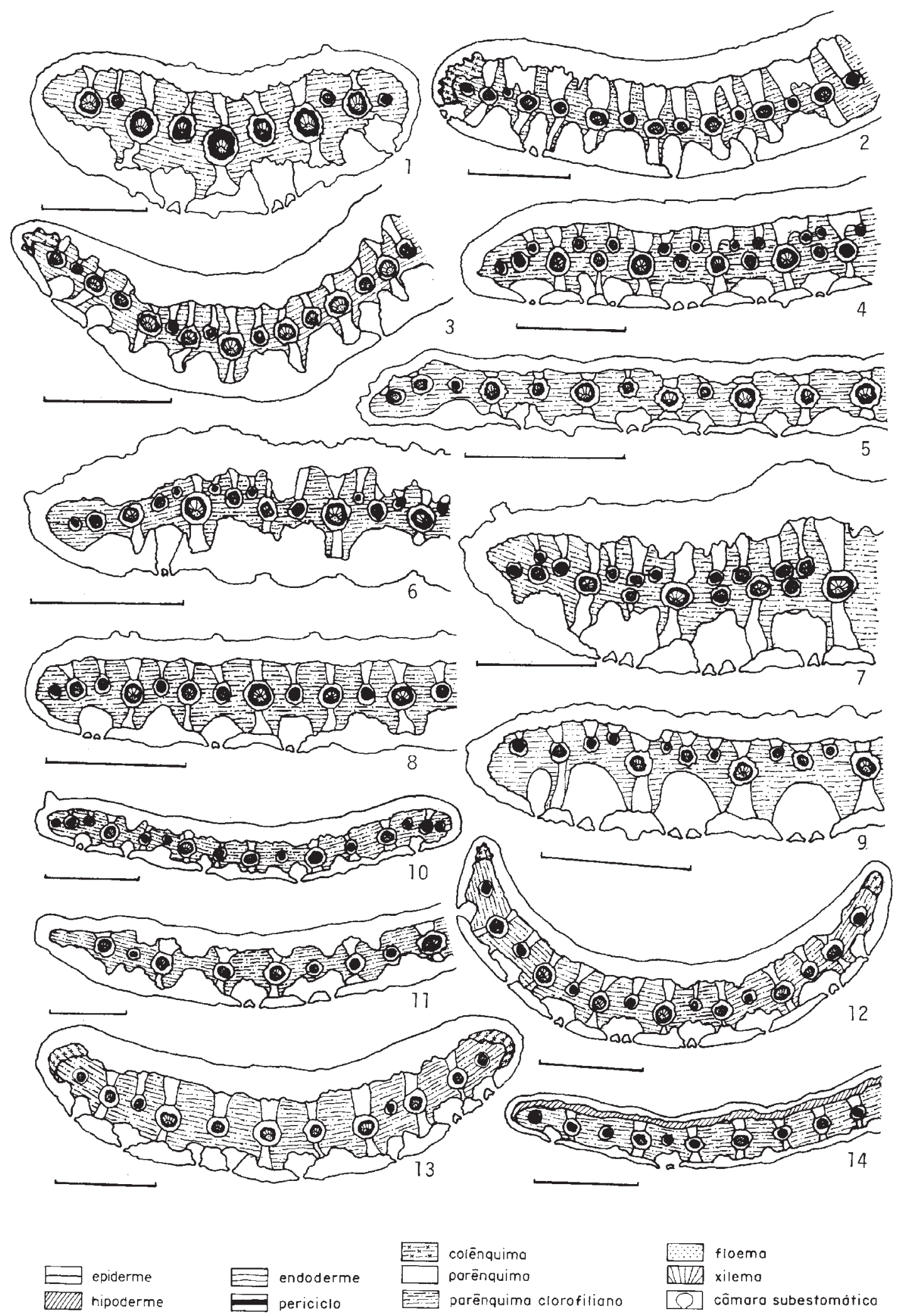

Figuras 1-14. Diagramas das secções transversais da região mediana das folhas e das brácteas de Actinocephalus. Folhas: 1. A. brachypus. 2. A stereophyllus. 3. A. denudatus. 4. A. polyanthus var. polyanthus. 5. A. diffusus. 6. Actinocephalus sp. nov. 3. 7. A. ciliatus. 8. A. polyanthus var. bifrons. 9. A. callophyllus. Brácteas: 10. Actinocephalus sp. nov. 1. 11. Actinocephalus sp. nov. 2. 12. A. cabralensis. 13. A. falcifolius. 14. A. claussenianus. Barras $=400 \mu \mathrm{m}(1,10,14), 600 \mu \mathrm{m}(2,5,8), 550 \mu \mathrm{m}(3,9)$, $700 \mu \mathrm{m}(4,6), 450 \mu \mathrm{m}(7,12-13), 300 \mu \mathrm{m}(11)$. 
As folhas e as brácteas de todos os espécimes estudados apresentam tricomas e em corte transversal evidenciam-se as células basal e colar dos mesmos, como pode ser observado em A. brachypus (figura 15) e em $A$. polyanthus var. polyanthus (figura 23 ).

Em A. cabralensis (figura 18) e em A. falcifolius (figura 19) observa-se a presença de colênquima situado logo abaixo da epiderme, nas margens das folhas.

Em todas as folhas e brácteas dos espécimes estudados o mesofilo é constituído de parênquima clorofiliano frouxo e é descontínuo devido à presença de extensões de bainha dos feixes vasculares, que são constituídas por células parenquimáticas alongadas no sentido longitudinal, em secção transversal (figuras 15-23).

Os feixes vasculares do mesofilo são sempre colaterais e envolvidos por bainha dupla (figuras 15-23). A bainha externa (células parenquimáticas) é a endoderme e a bainha interna (células de paredes espessadas) é o periciclo (figuras 15, 22).

Escapos - Os escapos dos diferentes espécimes estudados de Actinocephalus, em secção transversal da região mediana, estão representados nos diagramas das figuras 25-35. Todos os escapos estudados são cilíndricos e apresentam epiderme, córtex e cilindro vascular. A maioria dos escapos apresenta simetria radiada (figuras 25, 27-35), com exceção de A. polyanthus var. polyanthus (Sano et al. 142) e Actinocephalus sp. nov. 3 (figura 26), que apresentam simetria bilateral.

Todos os escapos apresentam de duas a cinco costelas, que formam saliências em A. claussenianus (figura 25), Actinocephalus sp. nov. 2 (figura 29) e A. bongardii (figura 35 ). O número de costelas pode variar entre os indivíduos de diferentes populações, como pode ser observado em A. herzogii (Ganev 285; Queiroz H51527) (figuras 31-32), e inclusive no mesmo indivíduo, como pode ser observado em $A$. bongardii (CFCR 1731) (figuras 34-35). Todos os escapos apresentam endoderme descontínua, que forma calotas nos feixes vasculares menores (figuras 25-35). O periciclo é contínuo e sinuoso, circundando totalmente os feixes vasculares menores e parcialmente os feixes vasculares maiores (figuras 25-35).
Os feixes vasculares são colaterais, de dois tamanhos distintos: os maiores voltados ao colênquima ou parênquima e os menores voltados ao parênquima clorofiliano (figuras 25-35). Foi encontrado feixe vascular composto apenas em A. herzogii (Queiroz H51527) (figuras 32, 38) e feixe vascular geminado apenas em A. bongardii (CFCR 1731) (figura 35).

A epiderme dos escapos de todos os espécimes estudados é unisseriada (figuras 36-40). Apresenta células com parede periclinal externa mais espessada que a interna, como em A. denudatus (figura 36), A. rigidus (figura 37), A. herzogii (Queiroz H51527) (figura 38), Actinocephalus sp. nov. 3 (figura 39), ou espessamento homogêneo, como em A. ciliatus (CFCR 11933) (figura 40).

Os estômatos estão situados em frente ao parênquima clorofiliano e possuem câmaras subestomáticas amplas (figuras 37, 39).

O córtex pode ser constituído por colênquima, como em A. denudatus (figura 36) e A. ciliatus (figura 40), ou por parênquima, como em A. herzogii (figura 38 ). O parênquima clorofiliano é frouxo (figuras 36,38 ); a endoderme é descontínua e constituída por células de paredes finas, celulósicas (figuras 36, 38-40). O periciclo é contínuo, constituído por células de paredes espessadas lignificadas, como pode ser observado em A. denudatus (figura 36) e em Actinocephalus sp. nov. 3 (figura 39). Os feixes vasculares colaterais apresentam elementos de metaxilema bem desenvolvidos (figura 36). A medula é constituída por células parenquimáticas em todos os espécimes estudados (figuras 38, 40).

\section{Discussão}

As folhas, as brácteas e os escapos de Actinocephalus (Koern.) Sano apresentam células epidérmicas com paredes parcialmente espessadas. Tal espessamento pode ser homogêneo ou diferencial, sendo a parede periclinal externa mais espessada que a interna. Essa característica, juntamente com a presença de tecido de sustentação na margem das folhas e das brácteas de algumas espécies, bem como no córtex de alguns escapos, está relacionada ao ambiente típico de campos rupestres a que estas plantas estão sujeitas.

Figures 1-14. Diagrams of the median cross sections of Actinocephalus leaves and bracts. Leaves: 1. A. brachypus. 2. A. stereophyllus. 3. A. denudatus. 4. A. polyanthus var. polyanthus. 5. A. diffusus. 6. Actinocephalus sp. nov. 3. 7. A. ciliatus. 8. A. polyanthus var. bifrons. 9. A. callophyllus. Bracts: 10. Actinocephalus sp. nov. 1. 11. Actinocephalus sp. nov. 2. 12. A. cabralensis. 13. A. falcifolius. 14. A. claussenianus. Bars $=400 \mu \mathrm{m}(1,10,14), 600 \mu \mathrm{m}(2,5,8), 550 \mu \mathrm{m}(3,9), 700 \mu \mathrm{m}(4,6)$, $450 \mu \mathrm{m}$ (7, 12-13), $300 \mu \mathrm{m}$ (11). 

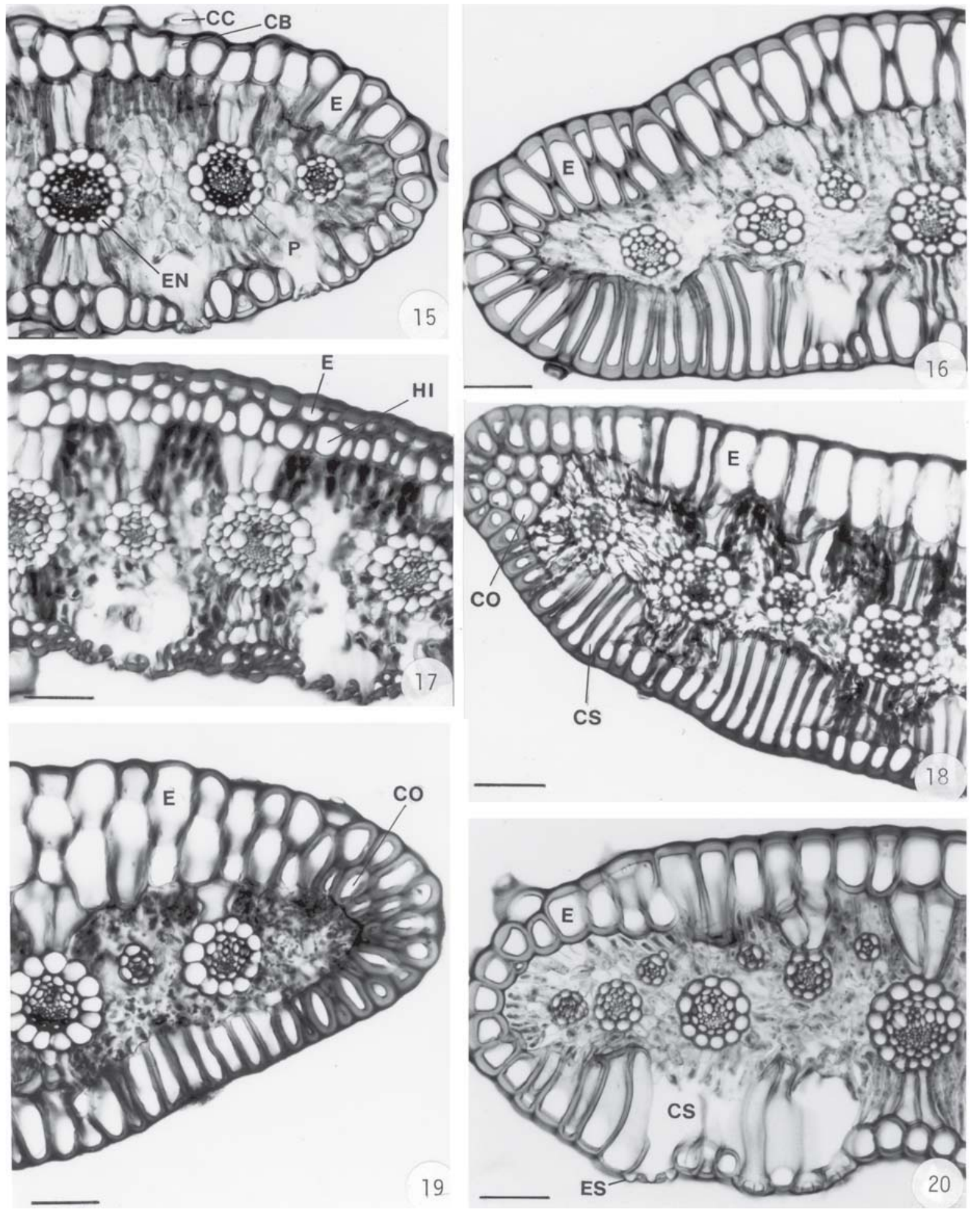

Figuras 15-20. Secções transversais da região mediana das folhas de Actinocephalus: 15. A. brachypus (CFCR 2673). 16. A. robustus. 17. A. claussenianus. 18. A. cabralensis. 19. A. falcifolius. 20. A. polyanthus var. bifrons (Scatena et al. 244). Barras $=80 \mu \mathrm{m}(15-20)$. $(\mathrm{CB}=$ célula basal $; \mathrm{CC}=$ célula colar; $\mathrm{CO}=$ colênquima; $\mathrm{CS}=$ câmara subestomática; $\mathrm{E}=$ epiderme; $\mathrm{EN}=$ endoderme; $\mathrm{ES}=$ estômato; $\mathrm{P}=$ periciclo).

Figures 15-20. Median cross sections of Actinocephalus leaves: 15. A. brachypus (CFCR 2673). 16. A. robustus. 17. A. claussenianus. 18. A. cabralensis. 19. A. falcifolius. 20. A. polyanthus var. bifrons (Scatena et al. 244). Bars $=80 \mu \mathrm{m}$ (15-20). $(\mathrm{CB}=$ basal cell $; \mathrm{CC}=$ colar cell $; \mathrm{CO}=$ collenchyma; $\mathrm{CS}=$ substomatal chamber; $\mathrm{E}=$ epidermis; $\mathrm{EN}=$ endodermis; $\mathrm{ES}=$ stoma; $\mathrm{P}=$ pericycle). 

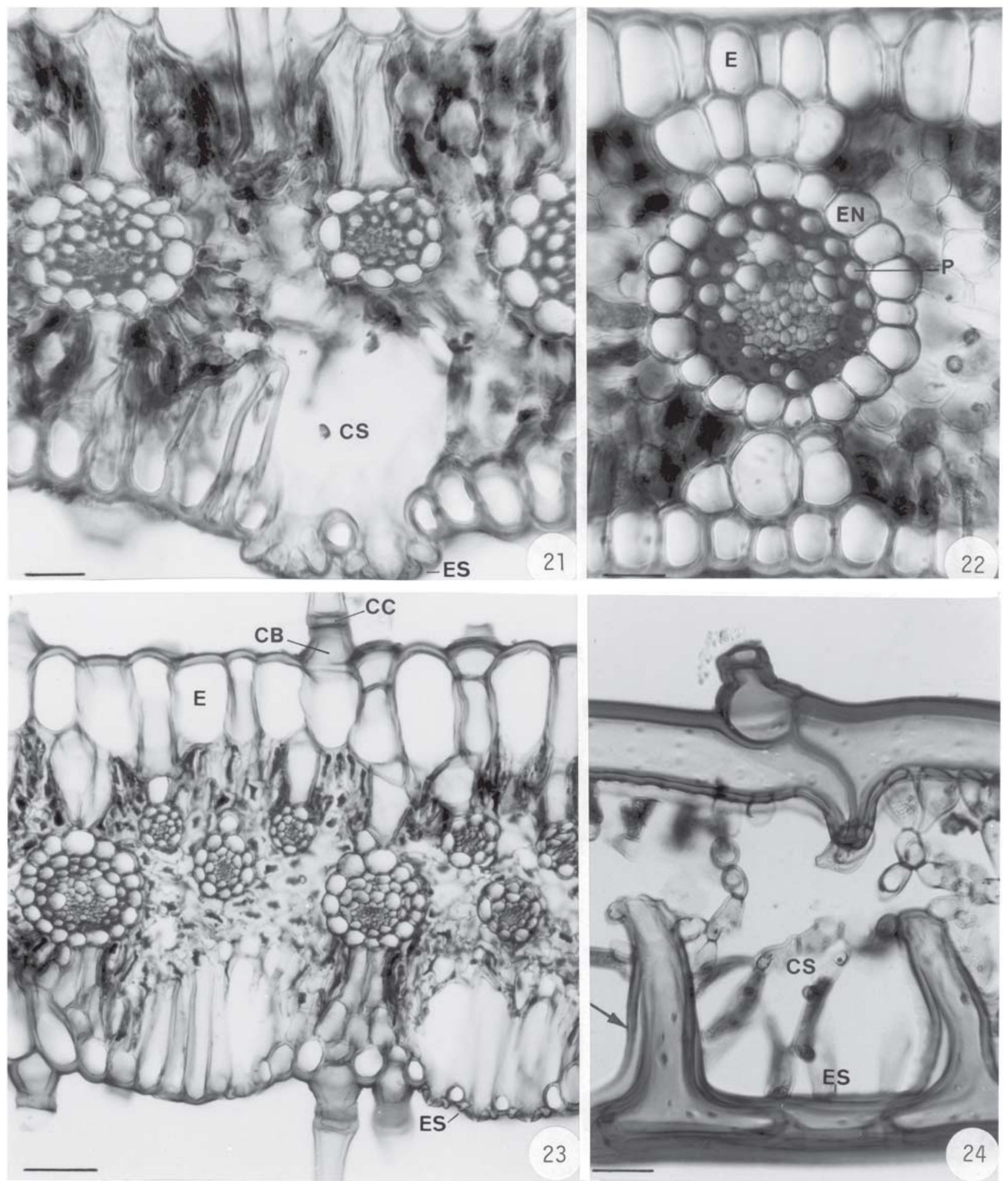

Figuras 21-24. Secções transversais e longitudinal da região mediana das brácteas de Actinocephalus. Transversais: 21. A. brachypus (Braga \& Chaves 446). 22. A. polyanthus var. polyanthus (Scatena et al. 237). 23. A. polyanthus var. polyanthus (Sano et al. 142). Longitudinal: 24. A. ramosus (Scatena et al. 249). Barras $=30 \mu \mathrm{m}(21-22,24), 80 \mu \mathrm{m}(23)$. ( $\mathrm{CB}$ = célula basal; $\mathrm{CC}$ = célula colar; $\mathrm{CS}=$ câmara subestomática; $\mathrm{E}=$ epiderme; $\mathrm{EN}$ = endoderme; $\mathrm{ES}$ = estômato; $\mathrm{P}=$ periciclo).

Figures 21-24. Median cross and longitudinal sections of Actinocephalus bracts. Cross sections: 21. A. brachypus (Braga \& Chaves 446). 22. A. polyanthus var. polyanthus (Scatena et al. 237). 23. A. polyanthus var. polyanthus (Sano et al. 142). Longitudinal section: 24. A. ramosus (Scatena et al. 249). Bars $=30 \mu \mathrm{m}(21-22,24), 80 \mu \mathrm{m}$ (23). (CB = basal cell; $\mathrm{CC}=$ colar cell; $\mathrm{CS}=$ substomatal chamber; $\mathrm{E}=$ epidermis; $\mathrm{EN}=$ endodermis $; \mathrm{ES}=$ stoma; $\mathrm{P}=$ pericycle $)$. 
Tais características podem proteger folhas, brácteas e escapos contra a transpiração e luminosidade excessivas, além de funcionarem como suporte mecânico contra a ação dos ventos, que são constantes (Scatena \& Menezes 1996), estando presentes também em folhas de outras famílias representativas de campos rupestres, como Compositae (Handro et al. 1970, Sajo \& Menezes 1994), Velloziaceae (Mello-Silva 1990), Iridaceae (Chueiri-Chiaretto 1984) e Xyridaceae (Sajo et al. 1995).

Segundo Rizzini (1976), o desenvolvimento dos tecidos mecânicos acha-se estreitamente ligado às intensidades luminosas, sendo que o espessamento das paredes celulares acompanha o incremento da luz e os caracteres heliomórficos decorrentes exibem proporcionalidade com aquela. Assim, tais características podem ser entendidas como heliomorfoses, dada a intensa radiação solar a que tais plantas estão sujeitas.

Quanto à disponibilidade de água, as características anatômicas observadas em Actinocephalus não correspondem a adaptações estruturais presentes em plantas xerófilas ou hidrófilas e, por isso, são mais propriamente relacionáveis a um ambiente mésico. Isto corrobora aquilo que é verificado na natureza, já que a grande maioria das espécies de Actinocephalus estudadas encontra-se sempre associada a linhas de drenagem ou a pequenos vales com solo úmido e encharcado na maior parte do ano.

Um aspecto notável na região dos estômatos é a presença de células altas na epiderme intercaladas com células curtas, em secção transversal, dependendo do nível em que passa o corte. Isto se deve pelo formato de "T invertido" das células que circundam os estômatos, quando observadas em secção longitudinal, semelhante ao que ocorre nas espécies de Paepalanthus, estudadas por Castro e Menezes (1990). O conjunto dessas células delimita as amplas câmaras subestomáticas, presentes nas folhas, nas brácteas e nos escapos de Actinocephalus.

Segundo Fahn \& Cutler (1992), as câmaras subestomáticas amplas estabelecem um longo gradiente de difusão entre o parênquima clorofiliano e o meio ambiente, além de alta resistência à difusão, contribuindo, mesmo quando os estômatos estão totalmente abertos, para baixas taxas de transpiração. Concordando com Scatena \& Rocha (1995), aceita-se que a presença de câmaras subestomáticas seja uma característica anatômica importante para a taxonomia das Eriocaulaceae, uma vez que já foi descrita para grupos taxonômicos distintos dentro da família, como algumas espécies de Paepalanthus (Castro \& Menezes 1990, Scatena \& Barros 1996, Scatena \& Rosa 2001), Syngonanthus (Scatena \& Menezes 1993, 1996) e Leiothrix (Scatena \& Rocha 1995, Scatena \& Giulietti 1996), embora com padrões anatômicos diferentes.

Feixes vasculares foliares envolvidos por bainha dupla nas folhas são considerados característicos para Eriocaulaceae (Tomlinson 1969, Monteiro et al. 1984, 1985, Castro \& Menezes 1995, Scatena \& Menezes 1996, Scatena et al. 1999b, Coan et al. 2002). Conforme apresentado por Scatena \& Menezes (1996) para espécies de Syngonanthus, por homologia, a bainha externa tem origem endodérmica e a bainha interna tem origem pericíclica.

Apesar da variação anatômica observada nos órgãos estudados de Actinocephalus, como espessamento das células epidérmicas e o número de feixes vasculares nas folhas, nas brácteas e nos escapos; margem das folhas e das brácteas; número de costelas e tecido que constitui o córtex nos escapos, Actinocephalus é bem caracterizado por algumas estruturas anatômicas comuns a todas as espécies estudadas. Nas folhas e nas brácteas tais estruturas são: epiderme unisseriada com células alongadas no sentido longitudinal, estômatos somente na face abaxial, câmaras subestomáticas amplas, feixes vasculares colaterais envolvidos por bainha dupla e extensões de bainha dos feixes vasculares constituídas por células parenquimáticas alongadas. Já nos escapos, podemos encontrar epiderme unisseriada, câmaras subestomáticas amplas, parênquima clorofiliano frouxo, endoderme descontínua, periciclo sinuoso, feixes vasculares colaterais e medula parenquimática.

Assim, com base nos resultados apresentados no presente trabalho e face à profunda diversidade de caracteres em Paepalanthus, pode-se afirmar que Actinocephalus apresenta marcada homogeneidade nos aspectos anatômicos, como também foi observado por Sano (P.T. Sano, dados não publicados) com relação aos aspectos morfológicos.

A presença de epiderme unisseriada com tricomas e feixes vasculares colaterais nas folhas, nas brácteas e nos escapos, feixes vasculares envolvidos por bainha dupla nas folhas e nas brácteas, medula parenquimática e periciclo sinuoso nos escapos são características típicas das Eriocaulaceae em geral, ocorrendo em todas as espécies já estudadas, pertencentes a: Paepalanthus (Castro \& Menezes 1995, Scatena \& Barros 1996, Scatena \& Moraes 1996, Scatena et al. 1998, Scatena \& Rosa 2001, Coan et al. 2002), Eriocaulon (Monteiro et al. 1984, Scatena et al. 1999a, Coan et al. 2002), 

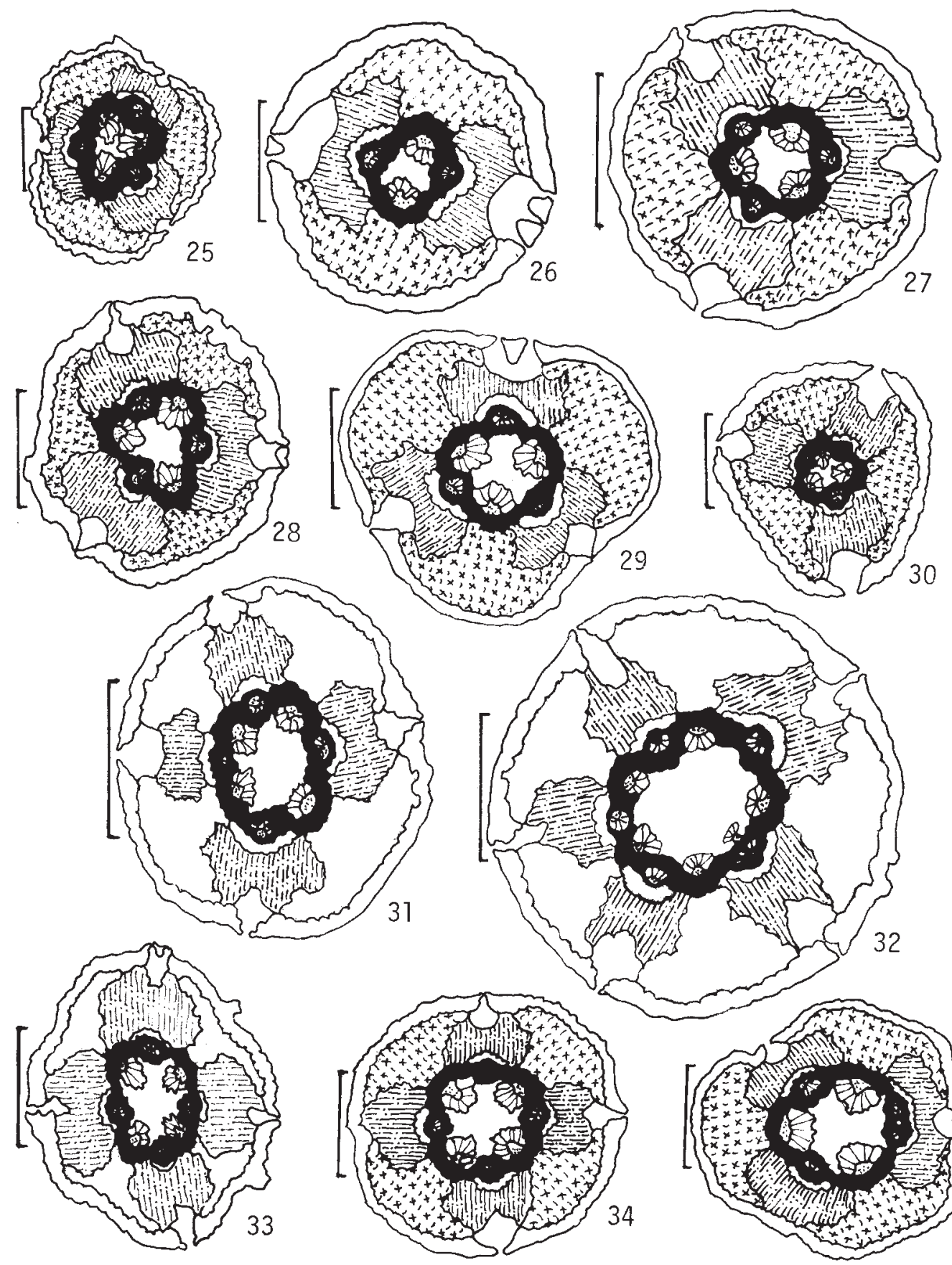

34
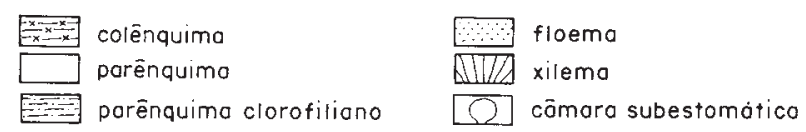

Figuras 25-35. Diagramas das secções transversais da região mediana dos escapos de Actinocephalus: 25. A. claussenianus. 26. Actinocephalus sp. nov. 3. 27. A. ciliatus. 28. A. brachypus (CFCR 8640). 29. Actinocephalus sp. nov. 2. 30. A. callophyllus. 31. A. herzogii (Ganev 285). 32. A. herzogii (Queiroz H51527). 33. A. brachypus (CFCR 2334). 34-35. A. bongardii (CFCR 1731). Barras $=150 \mu \mathrm{m}(25), 250 \mu \mathrm{m}(26-27,29), 220 \mu \mathrm{m}(28,33-35), 180 \mu \mathrm{m}$ (30), $300 \mu \mathrm{m}(31-32)$.

Figures 25-35. Diagrams of the median cross sections of Actinocephalus scapes: 25. A. claussenianus. 26. Actinocephalus sp. nov. 3. 27. A. ciliatus. 28. A. brachypus (CFCR 8640). 29. Actinocephalus sp. nov. 2. 30. A. callophyllus. 31. A. herzogii (Ganev 285). 32. A. herzogii (Queiroz H51527). 33. A. brachypus (CFCR 2334). 34-35. A. bongardii (CFCR 1731). Bars $=150 \mu \mathrm{m}(25)$, $250 \mu \mathrm{m}(26-27,29), 220 \mu \mathrm{m}(28,33-35), 180 \mu \mathrm{m}(30), 300 \mu \mathrm{m}(31-32)$. 

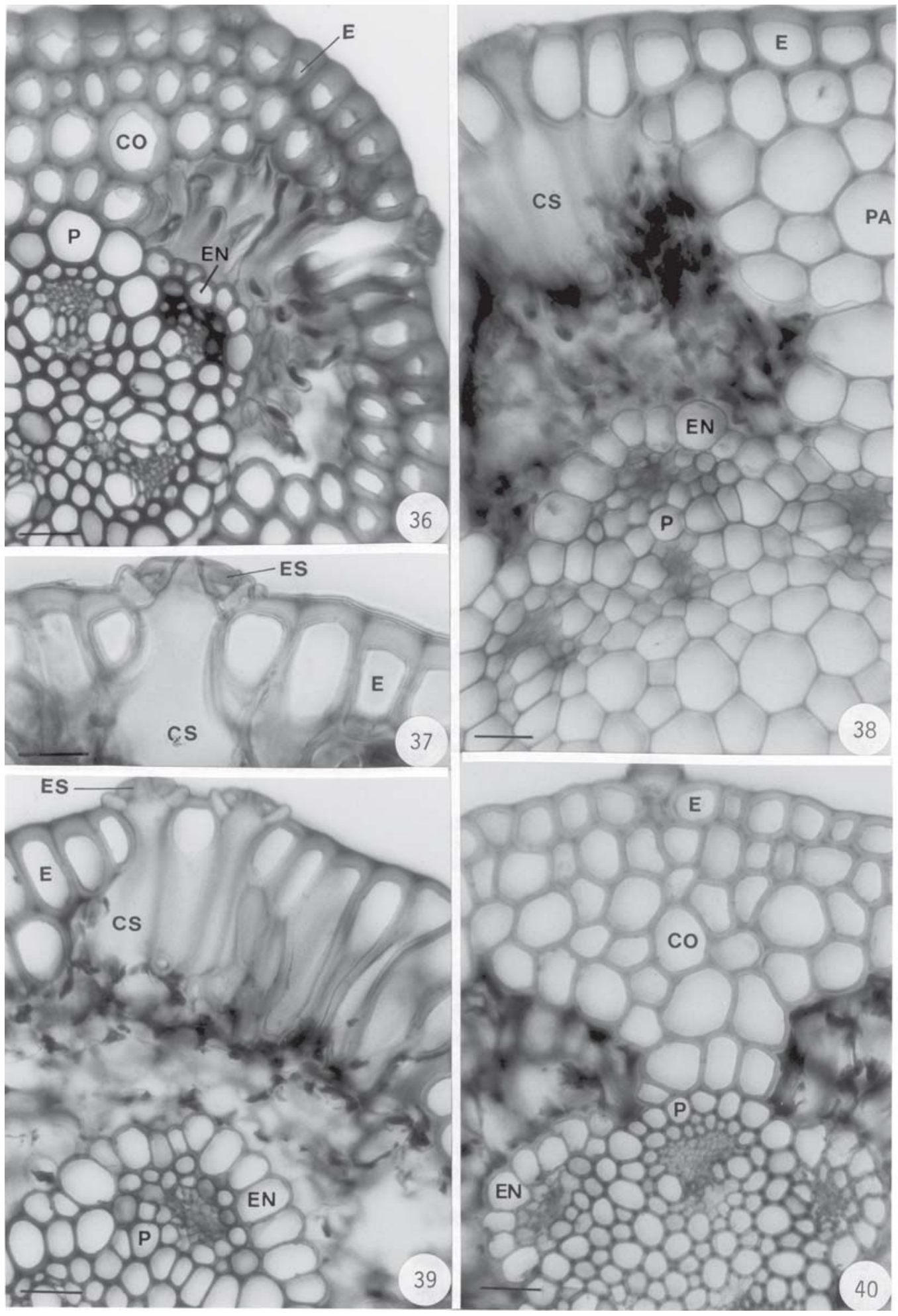

Figuras 36-40. Secções transversais da região mediana dos escapos de Actinocephalus: 36. A. denudatus. 37. A. rigidus. 38. A. herzogii (Queiroz H51527). 39. Actinocephalus sp. nov. 3. 40. A. ciliatus (CFCR 11933). Barras $=20 \mu \mathrm{m}$ (37), $30 \mu \mathrm{m}$ (36, 38-40). ( $\mathrm{CO}=$ colênquima; $\mathrm{CS}$ = câmara subestomática; $\mathrm{E}$ = epiderme; $\mathrm{EN}$ = endoderme; $\mathrm{ES}=$ estômato; $\mathrm{P}$ = periciclo; $\mathrm{PA}=$ parênquima).

Figures 36-40. Median cross sections of Actinocephalus scapes: 36. A. denudatus. 37. A. rigidus. 38. A. herzogii (Queiroz H51527). 39. Actinocephalus sp. nov. 3. 40. A. ciliatus (CFCR 11933). Bars $=20 \mu \mathrm{m}$ (37), $30 \mu \mathrm{m}(36,38-40)$. (CO = collenchyma; $\mathrm{CS}=$ substomatal chamber; $\mathrm{E}=$ epidermis; $\mathrm{EN}=$ endodermis; $\mathrm{ES}=$ stoma; $\mathrm{P}=$ pericycle; $\mathrm{PA}=$ parenchyma). 
Blastocaulon (Scatena et al. 1999b), Leiothrix (Monteiro et al. 1985, Scatena \& Rocha 1995, Scatena \& Giulietti 1996, Giulietti et al. 1998, Coan et al. 2002), Syngonanthus (Scatena \& Menezes 1996, Coan et al. 2002), Tonina e Philodice (Coan et al. 2002).

Já a presença de células alongadas constituindo tanto a epiderme como as extensões de bainha dos feixes vasculares nas folhas e nas brácteas, assim como endoderme descontínua nos escapos e câmara subestomática ampla nos três órgãos estudados são consistentes para o grupo e corroboram a proposta do novo gênero, Actinocephalus (Koern.) Sano, elaborada por Sano (2004).

Embora alguns caracteres sejam variáveis e tenham sido interpretados como respostas adaptativas às condições ambientais a que os táxons estudados estão sujeitos, a maioria das características anatômicas observadas em Actinocephalus são muito semelhantes às características observadas em Paepalanthus subsect. Aphorocaulon Ruhl., estudadas por Scatena \& Rosa (2001), e por isso devem decorrer muito mais de um provável parentesco do que só do ambiente. Isto corrobora os resultados de Giulietti et al. (2000), que apresentam uma árvore de consenso estrito para dados anatômicos e macromorfológicos, confirmando o polifiletismo de Paepalanthus e o monofiletismo de Paepalanthus sect. Actinocephalus Ruhl., tendo como grupo-irmão Paepalanthus subsect. Aphorocaulon.

As características anatômicas observadas em Actinocephalus também contribuem para a conclusão de que Eriocaulaceae reflete os diferentes microambientes que ocorrem nos campos rupestres, sendo constituída por espécies com características notadamente xeromórficas, como as de Syngonanthus, estudadas por Scatena \& Menezes (1996), por espécies com características notadamente hidromórficas, como as espécies aquáticas estudadas por Coan et al. (2002), e por espécies com características notadamente mesomórficas, como as de Actinocephalus, estudadas neste trabalho.

Agradecimentos - À Fapesp, pela bolsa de Iniciação Científica concedida à primeira autora, e ao $\mathrm{CNPq}$, pela bolsa de pesquisa concedida à segunda autora.

\section{Referências bibliográficas}

CASTRO, N.M. \& MENEZES, N.L. 1990. Considerações sobre a natureza das células altas da epiderme abaxial das folhas de Paepalanthus Kunth. Boletim de Botânica da Universidade de São Paulo 12:95-100.
CASTRO, N.M. \& MENEZES, N.L. 1995. Aspectos da anatomia foliar de algumas espécies de Paepalanthus Kunth. Eriocaulaceae da Serra do Cipó (Minas Gerais). Acta Botanica Brasilica 9:213-229.

CHUEIRI-CHIARETTO, I. A. 1984. Estudos morfo-anatômicos da folha de espécies de Trimesia (Iridaceae). Hoehnea 11:47-58.

COAN, A.I., SCATENA, V.L. \& GIULIETTI, A.M. 2002. Anatomia de algumas espécies aquáticas de Eriocaulaceae brasileiras. Acta Botanica Brasilica 16:371-384.

FAHN, A. \& CUTLER, D.F. 1992. Xerophytes. Gebrüder Borntrager, Berlim.

GIULIETTI, A.M. \& PIRANI, J.R. 1988. Patterns of geographic distribution of some plant species from the Espinhaço Range, Minas Gerais and Bahia, Brazil. In Proceedings of a workshop on neotropical distribution patterns (P.E. Vanzolini \& W.R. Meyer, eds.). Academia Brasileira de Ciências, Rio de Janeiro, p.39-69.

GIULIETTI, A.M. \& HENSOLD, N. 1990. Padrões de distribuição geográfica dos gêneros de Eriocaulaceae. Acta Botanica Brasilica 4:133-159.

GIULIETTI, A.M., AMARAL, M.C.E. \& BITTRICH, V. 1995. Phylogenetic analysis of inter-and infrageneric relationships of Leiothrix Ruhland (Eriocaulaceae). Kew Bulletin 50:55-71.

GIULIETTI, A.M., SCATENA, V.L. \& CARDOSO, V.A. 1998. Anatomia de escapos e de folhas e sua aplicação na taxonomia de Leiothrix flavescens (Bong.) Ruhl. (Eriocaulaceae). Sitientibus 18:31-49.

GIULIETTI, A.M., SCATENA, V.L., SANO, P.T., PARRA, L., QUEIROZ, L.P., HARLEY, R.M., MENEZES, N.L., YSEPON, A.M.B., SALATINO, A., SALATINO, M.L., VILEGAS, W., SANTOS, L.C., RICCI, C.V., BONFIM, M.C.P. \& MIRANDA, E.B. 2000. Multidisciplinary studies on neotropical Eriocaulaceae. In Monocots: Systematics and evolution (K.L. Wilson \& D.A. Morrison, eds.). CSIRO Publishing, Collingwood, p.580-589.

HANDRO, W., CAMPOS, J.F.B.M. \& OLIVEIRA, Z.M. 1970. Sobre a anatomia foliar de algumas Compostas dos campos rupestres. Ciência e Cultura 22:107-126.

HOLM, T. 1901. Eriocaulon decangulare L. An anatomical study. Botanical Gazette 31:17-39.

JOLY, A.B. 1993. Botânica: Introdução à taxonomia vegetal. Editora Nacional, São Paulo.

MALMANCHE, L.A. 1919. Contribution à l'étude anatomique des Eriocaulacées et des families voisines. Restiacées, Centrolepidacées, Xyridacées, Philydracées, Mayacacées. Thesis, St. Cloud, Paris.

MELLO-SILVA, R. 1990. Morphological and anatomical differentiation of Vellozia hirsuta populations (Velloziaceae). Plant Systematics and Evolution 173:197-208. 
MONTEIRO, W.R., GIULIETTI,A.M. \& CASTRO, M.M. 1984. Aspects of leaf structure of some species of Eriocaulon L. (Eriocaulaceae) from Serra do Cipó (Minas Gerais, Brazil). Revista Brasileira de Botânica 7:137-147.

MONTEIRO, W.R., CASTRO, M.M. \& GIULIETTI, A.M. 1985. Aspects of leaf structure of some species of Leiothrix Ruhl. (Eriocaulaceae) from Serra do Cipó (Minas Gerais, Brazil). Revista Brasileira de Botânica 7:109-125.

POULSEN, V.A. 1888. Anatomiske studien over Eriocaulaceerne. Vidensk Meddr Dansk Naturhistorisk Foren 4:221-386.

RIZZINI, C.T. 1976. Tratado de fitogeografia do Brasil: Aspectos ecológicos. Editora da Universidade de São Paulo, São Paulo.

ROESER, K.R. 1962. Die Nadel der SchwarzkieferMassenprodukt und Kunstwert der Natur. Mikrokosmos 61:33-36.

RUHLAND, W. 1903. Eriocaulaceae. In Das Pflazenreich (A. Engler, ed.). Wilhelm Engelmann Leipzig, v.4, p.1-294.

SAJO, M.G. \& MENEZES, N.L. 1994. Considerações sobre a anatomia foliar de espécies de Vernonia Screb (Compositae) da Serra do Cipó (M.G.). Naturalia 19:173-183.

SAJO, M.G., WANDERLEY, M.GL. \& CARVALHO, L.M. 1995. Caracterização anatômica foliar para 14 espécies de Xyris L. (Xyridaceae) da Serra do Cipó, M.G. Acta Botanica Brasilica 9:101-114.

SANO, P.T. 2004. Actinocephalus (Koern.) Sano (Paepalanthus sect. Actinocephalus), a new genus of Eriocaulaceae, and other taxonomic and nomenclatural changes involving Paepalanthus Mart. Taxon 53:99-107.

SCATENA, V.L. \& BARROS, A.M. 1996. Aspectos morfoanatômicos de Paepalanthus hilairei Koern. (Eriocaulaceae) da Serra do Cipó, M.G. Arquivos de Biologia e Tecnologia 39:75-88.

SCATENA, V.L. \& GIULIETTI, A.M. 1996. The taxonomy and morphological and anatomical differentiation of populations of Leiothrix crassifolia (Eriocaulaceae). Plant Systematics and Evolution 199:243-258.
SCATENA, V.L. \& MENEZES, N.L. 1993. Considerações sobre a natureza da câmara subestomática e das células epidémicas das folhas de Syngonanthus Ruhl., seção Thysanocephalus Koern. (Eriocaulaceae). Revista Brasileira de Botânica 16:159-165.

SCATENA, V.L. \& MENEZES, N.L. 1996. Anatomia de escapos e folhas de Syngonanthus Ruhl. (Eriocaulaceae) de Campos Rupestres. Revista Brasileira de Biologia 56:317-332.

SCATENA, V.L. \& MORAES, A.R.S. 1996. Anatomia de raízes, folhas e escapos de Paepalanthus subgênero Platycaulon (Eriocaulaceae) da Serra do Cipó - MG. Arquivos de Biologia e Tecnologia 39:1021-1035.

SCATENA, V.L. \& ROCHA, C.L.M. 1995. Anatomia dos órgãos vegetativos e do escapo floral de Leiothrix crassifolia (Bong.) Ruhl. Eriocaulaceae, da Serra do Cipó - M.G. Acta Botanica Brasilica 9:195-211.

SCATENA, V.L. \& ROSA, M.M. 2001. Morphology and anatomy of the vegetative organs and scapes from Aphorocaulon (Paepalanthus, Eriocaulaceae). Brazilian Archives of Biology and Tecnology 44:49-58.

SCATENA, V.L., GIULIETTI, A.M. \& CARDOSO, V.A. 1998. Anatomia do escapo floral de espécies brasileiras de Paepalanthus subgênero Platycaulon (Eriocaulaceae). Acta Botanica Brasilica 2:121-133.

SCATENA, V.L., GIULIETTI, A.M. \& CARDOSO, V.A. 1999a. Anatomia de raízes, escapos e folhas de espécies de Eriocaulon L. (Eriocaulaceae). Boletim de Botânica da Universidade de São Paulo 18:11-20.

SCATENA, V.L., CARDOSO, V.A. \& GIULIETTI, A.M. 1999b. Morfoanatomia de espécies de Blastocaulon Ruhland (Eriocaulaceae). Acta Botanica Brasilica 13:29-41.

SPLETT, S., BARTHLOTT, W., STUTZEL, T. \& BARROS, M.A.G. 1993. Leaf anatomy of brazilian Eriocaulaceae and its diagnostic significance. Flora 188:399-411.

TOMLINSON, P.B. 1969. Commelinales - Zingiberales. In Anatomy of the Monocotyledons III (C.R. Metcalfe, ed.). Clarendon Press, Oxford, v.3, p.1-446. 